\title{
QUALIDADE DE VIDA DE IDOSOS CADASTRADOS NA ESTRATÉGIA SAÚDE DA FAMÍLIA DE FOZ DO IGUAÇU-PR
}

\author{
Quality of life for elderly registered in the family health strategy (fhs) of Foz do \\ Iguaçu-PR
}

\section{Calidad de vida en ancianos catastrados en la estrategia salud de la familia de Foz do Iguaçu-PR}

Jossiana Wilke Faller

Willian Augusto de Melo²

Gelena Lucinéia Gomes Silva Versa ${ }^{3}$

Sonia Silva Marcon ${ }^{4}$

\section{RESUMO}

Estudo transversal realizado com o objetivo de avaliar a qualidade de vida de idosos cadastrados nas Unidades de Saúde da Família (USF) de Foz do Iguaçu - PR. Os dados foram coletados no período de outubro de 2009 a março de 2010 na residência dos idosos, utilizando o WHOQOL-OLD. A amostra está constituída de 192 idosos selecionados aleatoriamente. Os resultados mostraram melhor qualidade de vida entre idosos mais jovens, brancos, com oito ou mais anos de estudo, que possuem companheiro, não moram sozinhos, seguem uma religião, exercem atividade remunerada e não são aposentados. 0 envelhecimento e a morte são vistos como fato natural, e o aspecto que mais compromete a qualidade de vida dos idosos é a falta de perspectivas de participação social na comunidade. Conclui-se pela necessidade dos profissionais de saúde de atentarem mais para os aspectos que influenciam negativamente na determinação da qualidade de vida dos idosos.

Palavras-chave: Qualidade de Vida. Envelhecimento. Assistência a Idosos. Enfermagem. Promoção da Saúde.

\begin{abstract}
A cross-sectional study accomplished with the objective of evaluating the quality of life for elderly registered in the Family Health Units of Foz do Iguaçu - PR. The data were collected from October 2009 to March 2010 in the seniors' residence, using WHOQOL-OLD. The sample consists of 192 seniors selected randomly. The results showed better life quality among not so old seniors, white, with eight or more years of study, living with a partner, that had a religious faith, had a paid work, and that are not retired yet. Aging and death are seen as a natural fact and the aspect that jeopardizes the most the quality of the seniors' life is the lack of perspectives of social participation in the community. It is concluded that there is a need for health professionals to pay more attention to the aspects that influence negatively in the determination of the quality of the seniors' life.
\end{abstract}

Keywords: Quality of Life. Aging. Old Age Assistance. Nursing. Health Promotion.

\section{Resumen}

Estudio transversal realizado con el objetivo de evaluar la calidad de vida de ancianos inscritos en las Unidades de Salud de la Familia (USF) de Foz do Iguaçu - PR. Los datos fueron recolectados en el período de octubre de 2009 a marzo de 2010 en la residencia de los ancianos, utilizando el WHOQOL-OLD. La muestra está constituida de 192 ancianos seleccionados al azar. Los resultados mostraron mejor calidad de vida entre ancianos más jóvenes, blancos, con ocho o más años de estudio, que poseen compañero, que no viven solos, siguen una religión, ejercen actividad remunerada y no son jubilados. El envejecimiento y la muerte son vistos como hecho natural y el aspecto que más compromete la calidad de vida de los ancianos es la falta de perspectivas de participación social en la comunidad. Se concluye que hay una necesidad de que los profesionales de salud se atenten más para los aspectos que influencian negativamente en la determinación de la calidad de vida de los ancianos.

Palavras clave: Calidad de Vida. Envejecimiento. Asistencia a los Ancianos. Enfermería. Promoción de la Salud.

'Enfermeira. Mestranda em Enfermagem pela Universidade Estadual de Maringá (UEM). Maringá - PR. Docente do curso de Enfermagem da Universidade Estadual do Oeste do Paraná (UNIOESTE). Foz do Iguaçu - PR. Brasil. E-mail: jofaller@hotmail.com..'Enfermeiro. Mestre em Enfermagem. Docente do Curso de Enfermagem do Centro Universitário de Maringá (CESUMAR). Maringá- PR. Brasil. E-mail: profewill@yahoo.com.br. ${ }^{3}$ Enfermeira. Mestranda em Enfermagem pela Universidade Estadual de Maringá (UEM). Maringá - PR. Coordenadora das Comissões Intra-hospitalares do Hospital Universitário do Oeste do Paraná - HUOP. Cascavel - PR. Brasil. E-mail: gelenaenfermagem@yahoo.com.br, ${ }^{\circledR}$ Enfermeira. Doutora em Filosofia da Enfermagem. Professora da graduação e pós-graduação em Enfermagem na Universidade Estadual de Maringá. Maringá - PR. Brasil. E-mail: soniasilva.marcon@gmail.com 


\section{INTRODUCÃO}

0 crescimento da população idosa é um fenômeno mundial, e, no Brasil, as modificações se dão de forma radical e bastante acelerada. ${ }^{1}$ Com base no último censo realizado em $2000,8,6 \%$ da população brasileira é constituída por idosos. As projeções mais conservadoras indicam que, em 2020, já seremos o sexto país do mundo em número de idosos, com um contingente superior a 30 milhões de pessoas, com o grupo etário de maior crescimento o de 75 anos ou mais, revelando $49,3 \%{ }^{2}$

A velocidade deste processo traz uma série de questões cruciais, como o aumento de gastos na saúde devido às doenças crônicas e enfermidades complexas típicas da idade, que perduram por longos anos, exigindo cuidados contínuos, atendimentos especializados e internações.

Esta realidade nos alerta para o fato de que quanto mais idosos, mais precisaremos de políticas públicas que permitam um envelhecimento saudável à população, ou seja, viver mais e melhor. ${ }^{3}$

A falta de valorização das aposentadorias e pensões, a precariedade de investimentos públicos, a desinformação, o preconceito, o desrespeito, a falta de instalações adequadas, a carência de programas específicos e mesmo de recursos humanos, trazem medo e angústia aos idosos brasileiros, sendo contribuintes para um envelhecimento não saudável, interferindo direta ou indiretamente na qualidade de vida do idoso. ${ }^{4}$

0 homem em seu desenvolvimento durante o ciclo da vida é um ser biopsicossocial, podendo sofrer influências e influenciar o ambiente em que vive num processo de adaptação em suas relações com o mundo. Este ambiente pode facilitar ou dificultar o processo de adaptação, acelerando ou retardando o envelhecimento. ${ }^{5}$

0 processo de envelhecimento, por sua vez, pode ser definido a partir de alterações no organismo, demonstradas de forma variável e individual. Também pode ser caracterizado por um processo dinâmico, progressivo e irreversível, no qual interagem múltiplos fatores biológicos, psíquicos e sociais, acompanhado por alterações significativas na composição, estrutura e fisiologia dos sistemas orgânicos e do indivíduo como um todo. Esse processo ainda pode ser acompanhado e marcado por mudanças na composição familiar e doméstica, como a viuvez e problemas de ordem econômica, como aposentadorias insatisfatórias, que, somadas a outros fatores psicossociais como solidão e baixa autoestima, ocasionam mudanças no estado de saúde de cada idoso. ${ }^{6}$

Envelhecer com qualidade de vida é uma preocupação entre os profissionais da área da saúde. Mas não é fácil definir qualidade de vida para os idosos, havendo várias discussões sobre este conceito. ${ }^{7}$

De acordo com a Organização Mundial de Saúde (OMS), a qualidade de vida (QV) é definida como: "[...] a percepção que o indivíduo tem de sua posição na vida dentro do contexto de sua cultura e do sistema de valores de onde vive, e em relação a seus objetivos, expectativas, padrões e preocupações". ${ }^{8} 0$ grupo de estudiosos sobre QV da OMS World Health Organization Quality of Life (WHOQOL-Group) propõe um conceito para QV subjetivo, multidimensional e que inclui elementos positivos e negativos. ${ }^{9}$

O instrumento elaborado por este grupo para avaliação da QV, o WHOQ0L-100, é adequado e pertinente para medir a qualidade de vida em adultos mais velhos, mas não suficientemente abrangente. Há aspectos fundamentais na composição da qualidade de vida em adultos mais velhos que não estão incluídos neste instrumento (e, consequentemente, no WHOQOL-BREF, uma vez que este é uma versão condensada do primeiro). ${ }^{10}$

Contudo, a necessidade de instrumentos de rápida aplicação determinou o desenvolvimento da versão WHOQOLOLD (World Health Organization Quality of Life Assessment for Older Adults) utilizado para avaliar a qualidade de vida em adultos mais velhos, fornecendo informações adicionais desta população específica. ${ }^{10}$

A investigação da qualidade de vida relacionada à saúde identifica aspectos em relação às potencialidades e peculiaridades de saúde e vida do idoso que podem interferir no processo saúde-doença. ${ }^{11}$

Desta forma, o objetivo do estudo foi avaliar a qualidade de vida de idosos cadastrados nas unidades de saúde da familia (USF) e conhecer as características sociodemográficas desta população.

\section{TRAJETÓRIA METODOLÓGICA}

Estudo descritivo-exploratório, do tipo observacional, com delineamento transversal, realizado no município de Foz do Iguaçu, região oeste do estado do Paraná. Este município tem uma população de 325.132 habitantes, dos quais 21.665 são idosos, caracterizando 6,66\% da população.

$\mathrm{Na}$ área da saúde, o município conta com quatro hospitais, sendo apenas um público, 27 unidades básicas de saúde (UBS) e 32 equipes da Estratégia Saúde da Família (ESF), as quais cobrem $40 \%$ da população urbana. Esta estratégia cobre 36.771 famílias, 137.719 pessoas, sendo 10.803 acima de 60 anos.

Os participantes do estudo foram selecionados aleatoriamente a partir de dados dos prontuários dos idosos cadastrados nas USF localizadas nos cinco distritos sanitários do município (Sul, Nordeste, Sudeste, Centro-oeste e Norte). Os critérios de inclusão adotados foram: ter idade igual ou superior a 60 anos, considerada como etapa inicial do envelhecimento, segundo a Organização das Nações Unidas, para países em desenvolvimento como o Brasil (12); estar cadastrado em uma USF; estar consciente; não apresentar problemas cognitivos e/ou mentais e não estar institucionalizado.

0 tamanho da amostra a ser estudada foi calculado a partir do número total de indivíduos idosos cadastrados na ESF, 
com um erro de estimativa de $5 \%$ e confiabilidade de $95 \%$, acrescidos de $20 \%$ para possíveis perdas. Mediante a estratificação proporcional foi definido o número de indivíduos de cada distrito a serem incluídos no estudo. Além disso, apesar da diferença no número de idosos do sexo feminino e masculino, optou-se por uma composição igualitária entre os dois sexos.

Os dados foram coletados no período de outubro de 2009 a março de 2010, nos domićlios dos idosos, por meio de entrevista estruturada realizada por acadêmicos de enfermagem devidamente treinados para esta finalidade. As entrevistas tiveram uma duração média de 20 minutos, e as respostas foram registradas integralmente durante sua realização. Foram utilizados dois instrumentos: um questionário sociodemográfico e o questionário WHOQOL-OLD.

O questionário WHOQOL-OLDé constituído de 24 questões, do tipo escala de Likert de cinco pontos, divididos em seis facetas: ${ }^{13}$

a) Funcionamento do Sensório (FS) - avalia 0 funcionamento sensorial e o impacto da perda das habilidades sensoriais na qualidade vida;

b) Autonomia (AUT) - refere-se à independência na velhice, descrevendo até que ponto se é capaz de viver de forma autônoma e tomar suas próprias decisões;

c) Atividades passadas, presentes e futuras (PPF) descreve a satisfação sobre conquistas na vida e coisas a que se anseia;

d) Participação Social (PSO) - delineia a participação em atividades do cotidiano, especialmente na comunidade;

e) Morte ou Morrer (MM) - relacionam-se a preocupações, inquietações e temores sobre a morte e morrer;

f) Intimidade (INT) - avalia a capacidade de se ter relações pessoais e íntimas.

Cada uma das seis facetas está constituída por quatro itens, sendo que a soma dos itens que pertencem a uma mesma faceta produz o escore bruto da faceta (EBF), cujos valores (mínimo e máximo) são iguais em todas as facetas (amplitude de 4 a 20) e representa o mais baixo valor possível [número de itens (n) $x$ 1] e o mais alto valor possível [número de itens (n) $x$ 5] da respectiva faceta.

A comparação entre as facetas ocorre a partir de uma comparação dos escores brutos das facetas. Caso o EBF da faceta seja dividido pelo número de itens na faceta, o escore (médio) padronizado da faceta (EPF) pode ter qualquer valor decimal entre 1 e 5.0 valor 1 representa uma avaliação da qualidade de vida a mais baixa possível e o valor 5 uma avaliação da qualidade de vida a mais alta possível para o indivíduo; a transformação de um escore bruto para um escore transformado da escala (ETE) entre 0 e 100 possibilita expressar o escore da escala em percentagem entre o valor mais baixo possível (0) e o mais alto possível (100). ${ }^{13}$

A variável resposta ou desfecho foi a classificação da qualidade de vida dos idosos, a partir dos pontos de corte propostos pela OMS, sendo de 1,0 a 1,50 - ruim, de 1,51 até 2,50 - fraco, de 2,51 a 3,50 - regular, de 3,51 a 4,50 - bom e de 4,51 a 5,0 - ótimo. Ao analisar estatisticamente a associação entre as variáveis, optou-se por dicotomizar esta variável considerando como ponto de corte o escore médio da variável. Assim, para este estudo, a variável resposta ficou dicotomizada como "satisfatória" quando o escore foi e" 3 e "não satisfatória" quando inferior a 3.

As variáveis independentes do estudo foram categorizadas e dicotomizadas como segue: sexo (masculino e feminino), idade (faixa etária), estado civil (casado, solteiro, separado e viúvo/casado e não casado), escolaridade (analfabetos, ensino fundamental incompleto e completo, ensino médio incompleto e completo e ensino superior incompleto e completo/maior ou igual e menor que oito anos de estudo), etnia/cor (branca, negra, parda, indígena, amarela/branca e não branca), religião (católica, evangélica, muçulmana, espírita, não tem/praticante e não praticante), renda familiar (1 a 3 salários mínimos (SM), maior ou igual a 3 SM e menos de um SM), aposentado ou não, com atividade remunerada ou não, se paranaense ou não e com quem reside: Família Nuclear (FN), aquela constituída pelos cônjuges e filhos, Família Incompleta (FI) quando falta um dos membros da família nuclear; e Família Extensa (FE), quando formada por três ou mais gerações, e os que moram só.

Para análise, os dados coletados foram arquivados em planilha do Programa Excel, e para a realização das análises estatísticas foi utilizado o software Statística 7.1, constituída pelos testes do qui-quadrado de Pearson, para as variáveis que apresentavam tabela de contingência $3 \times 2$, o teste exato de Fisher, para as tabelas com variáveis dicotômicas ou com tabela de contingência $2 \times 2$, e o teste $V$ de Cramer para as tabelas que apresentaram frequência esperada valor zero ou menor que cinco em uma das caselas. Para todas as análises foram considerados o intervalo de confiança de $95 \%$ e o nível de significância de $5 \%$.

0 desenvolvimento do estudo obedeceu aos preceitos éticos disciplinados pela Resolução 196/96 do Conselho Nacional de Saúde e o projeto aprovado pelo Comitê Permanente de Ética em Pesquisa com Seres Humanos da Universidade do Oeste do Paraná - UNIOESTE (Parecer n. ${ }^{\circ} 463$ /2009/CEPE) e submetido à apreciação da Secretaria Municipal de Saúde do município de Foz do Iguaçu, para a devida liberação do campo de pesquisa.

\section{RESULTADOS E DISCUSSÃO}

Dos 192 idosos em estudo (oito foram excluídos por erro no preenchimento do instrumento de coleta de dados), $51 \%$ eram do sexo feminino, com predominância de idade na faixa etária de 60 a 69 anos (50,52\%), o que se justifica, em parte, as características do município, que se encontra em processo de envelhecimento populacional, atingindo na atualidade uma expectativa de vida de aproximadamente 71 anos, muito próximo da expectativa de vida no país, de 71,3 anos. ${ }^{14}$ Outro fator que pode ter contribuído para a predominância desta faixa etária é que os idosos mais velhos têm uma probabilidade maior de apresentarem doenças incompatíveis com os critérios de inclusão estabelecidos. Portanto, esta 
concentração de idosos neste grupo éuma característica do recente processo de envelhecimento brasileiro, diferente do que vem ocorrendo nos países desenvolvidos, onde a concentração é maior no grupo de 80 anos ou mais. ${ }^{12}$

A maioria dos indivíduos em estudo é casada (70,31\%), da cor branca $(63,54 \%)$ e com baixo grau de instrução - ensino fundamental incompleto $(68,2 \%)$. Segundo o último censo do IBGE de 2000, a média de escolaridade no estado do Paraná foi de 3,2 anos. Apesar dos avanços observados ao longo dos anos, a redução nas proporções de anaffabetismo está ocorrendo de forma muito lenta, eos idosos que possuem escolaridade mais alta ainda são escassos. ${ }^{2}$

A prática religiosa foi referida por $94,27 \%$ dos entrevistados, com predomínio da religião católica $(66,15 \%)$. Com o avançar da idade, a espiritualidade é fonte importante de suporte emocional, repercutindo nas áreas da saúde física e mental. Práticas e crenças religiosas contribuem para o bem-estar na velhice, exercendo grande influência nesta fase da vida. ${ }^{15}$

Em relação à renda, observou-se que a maioria dos idosos (79,01\%) tem umarenda de 1 a 3 salários mínimos, sendo que 17,24\% deles são aposentados que continuam exercendo alguma atividade remunerada. No Brasil, uma das características marcantes da população idosa é seu baixo poder aquisitivo. Aposentadorias e pensões constituem sua principal fonte de rendimento, e o retorno dos idosos ao mercado de trabalho ou a sua permanência se dá, sobretudo no mercado informal, em atividades mal remuneradas e jornadas de trabalho extensas. ${ }^{16}$

Uma parcela considerável dos idosos convive em uma família do tipo incompleta $(46,86 \%)$, e o número de moradores variou de 1 a 9 pessoas, prevalecendo os que residem com até 4 pessoas (53,12\%). Detalhes das características sociodemográficas estão demonstrados na Tabela 1.

Tabela 1 - Distribuição dos idosos em estudo, segundo variáveis sociodemográficas e a média global e desviopadrão dos pontos obtidos com a aplicação do WHOQOL-OLD. Foz do lguaçu - PR, 2010.

\begin{tabular}{|c|c|c|c|c|c|c|c|}
\hline VARIÁVEIS & $\mathbf{N}$ & $\%$ & $\begin{array}{c}\text { WHOQOL- } \\
\text { OLD } \\
\text { m } \pm d p \\
\end{array}$ & VARIÁVEIS & $\mathbf{N}$ & $\%$ & $\begin{array}{c}\text { WHOQOL- } \\
\text { OLD } \\
\text { m } \pm d p \\
\end{array}$ \\
\hline Sexo & & & & Religião & & & \\
\hline Feminino & 98 & 51,0 & $90 \pm 17,25$ & Católica & 127 & 66,1 & $86 \pm 13,04$ \\
\hline Masculino & 94 & 49,0 & $93 \pm 15,38$ & Evangélica & 52 & 27,1 & $84 \pm 9,93$ \\
\hline Idade & & & & Não tem & 10 & 5,2 & $82 \pm 11,41$ \\
\hline & & & & Muçulmano & 01 & 0,5 & 83 \\
\hline $60-69$ & 97 & 50,5 & $88 \pm 14,40$ & Espírita & 01 & 0,5 & 88 \\
\hline $70-79$ & 75 & 39,1 & $90 \pm 14,57$ & \multirow[t]{2}{*}{ Escolaridade } & & & \\
\hline $80-89$ & 19 & 9,9 & $89 \pm 13,61$ & & & & \\
\hline 90 e mais & 01 & 0,5 & $75 \pm 0$ & EFI & 131 & 68,2 & $83 \pm 11,85$ \\
\hline Etnia/cor & & & & $\mathrm{EFC}$ & 30 & 15,6 & $88 \pm 9,90$ \\
\hline & & & & EMI & 15 & 7,8 & $89 \pm 9,85$ \\
\hline Branco & 122 & 63,4 & $90 \pm 14,88$ & EMC & 08 & 4,8 & $98 \pm 10,45$ \\
\hline Negro & 40 & 20,8 & $86 \pm 14,42$ & Analfabetos & 05 & 2,6 & $76 \pm 16,17$ \\
\hline Pardo & 28 & 14,6 & $84 \pm 13,50$ & ESI & 03 & 1,6 & $97 \pm 18,25$ \\
\hline Indígena & 01 & 0,5 & 76 & \multirow[t]{2}{*}{ Estado Civil } & & & \\
\hline Amarelo & 01 & 0,5 & 01 & & & & \\
\hline \multirow[t]{2}{*}{ Renda } & & & & Casado & 135 & 70,3 & $92 \pm 12,08$ \\
\hline & & & & Viúvo & 40 & 20,8 & $79 \pm 10,95$ \\
\hline$-1 \mathrm{SM}$ & 18 & 9,4 & $86 \pm 13,75$ & Separado & 12 & 6,2 & $87 \pm 12,01$ \\
\hline 1 a $3 \mathrm{SM}$ & 152 & 79,8 & $85 \pm 11,68$ & Solteiro & 03 & 1,6 & $86 \pm 11,74$ \\
\hline + $3 \mathrm{SM}$ & 22 & 11,5 & $90 \pm 13,96$ & Divorciado & 02 & 1,0 & 85 \\
\hline Com q mora & & & & \multicolumn{3}{|l|}{$\mathbf{N}^{0}$ moradores } & \\
\hline Fam nuclear & 74 & 38,5 & $83,7 \pm 13,1$ & Um & 15 & 7,8 & $83,5 \pm 13,7$ \\
\hline Fam incomp & 90 & 46,9 & $84,0 \pm 13,1$ & Dois & 53 & 27,6 & $85,1 \pm 13,0$ \\
\hline Fam extensa & 13 & 6,78 & $83,7 \pm 13,1$ & Mais que dois & 124 & 64,6 & $84,0 \pm 13,0$ \\
\hline Sozinho & 15 & 7,81 & $83,5 \pm 13,7$ & \multirow[t]{2}{*}{ Naturalidade } & & & \\
\hline \multicolumn{4}{|l|}{ Nacionalidade } & & & & \\
\hline & & & & Paranaense & 70 & 36,5 & $83,9 \pm 13,1$ \\
\hline BR & 187 & 97,4 & $84,0 \pm 13,0$ & Outros estados & 122 & 63,5 & $84,1 \pm 13,1$ \\
\hline PY & 5 & 2,6 & $67,4 \pm 13,1$ & \multirow[t]{2}{*}{ Ativ Remun } & & & \\
\hline \multicolumn{4}{|l|}{ Aposentado } & & & & \\
\hline & & & & Sim & 64 & 33,3 & $84,0 \pm 13,1$ \\
\hline Sim & 146 & 76,0 & $84,0 \pm 13,0$ & \multirow[t]{2}{*}{ Não } & 128 & 66,7 & $84,0 \pm 13,0$ \\
\hline Não & 46 & 24,0 & $84,1 \pm 13,1$ & & & & \\
\hline
\end{tabular}

Escolaridade: FI - Fundamental Incompleto; FC - Fundamental Completo; EMI - Ensino Médio Incompleto; EMC - Ensino Médio Completo; ESI - Ensino Superior Incompleto;;Nacionalidade: BR - Brasileiros; PY - Paraguaios 
Os idosos do sexo masculino apresentaram melhores índices de QV em todas as facetas, exceto na faceta Intimidade. A faixa etária com melhor QV foi a de 60 a 69 anos, e esta cai drasticamente a cada década, de modo que menos da metade dos idosos com 80 ou mais anos apresentam índice de QV considerado adequado.

Melhores índices de QV também foram observados entre os idosos casados, de cor/etnia branca, nos que seguem uma religião, residem com a família, não aposentados, que exercem uma atividade remunerada, os de renda entre 1 a 3 salários mínimos e com escolaridade inferior a 8 anos.

Os idosos que moram com até duas pessoas demonstraram maior QV na faceta Autonomia e Intimidade. Os déficits de habilidades sensoriais que ocorrem no processo de envelhecimento afetam seriamente a QV; porém, estudos realizados com idosos demonstram não existir relação entre a queixa e o déficit dessas habilidades. Apesar de as perdas iniciarem em torno dos 30 anos, a maior parte dos indivíduos começa a apresentar as primeiras alterações audiométricas por volta dos 50 anos. A porcentagem de idosos que apresentam perda auditiva descrita na literatura é bastante variável, em torno de 32,2\% a $89,23 \% .^{17}$

A família constitui importante fonte de apoio em todas as circunstâncias da vida, importante ao seu bem-estar. No sexo masculino, os menores escores foram observados nos idosos que relataram viver só, e os maiores naqueles que residem em família incompleta. Cabe ressaltar, conforme dita a Política Nacional do Idoso, art. $3^{\circ}$, que a família, a sociedade e o Estado têm o dever de assegurar ao idoso todos os direitos da cidadania, garantindo sua participação na comunidade, defendendo sua dignidade, bem-estar e o direito à vida. ${ }^{18}$
0 fato de o idoso morar só é considerado uma situação de risco caso este possua algum comprometimento funcional. Contudo, morar só pode ser uma opção, possível se as condições financeiras e de saúde permitirem, vista a possibilidade de perda da autonomia e suporte familiar. A equipe de saúde deve estar atenta a estes idosos para verificar a existência de incapacidades, prever e prover recursos na comunidade para a continuidade do cuidado na ausência da família. ${ }^{6}$

Em relação ao estado civil, os valores significativos foram evidenciados nos casados em todas as facetas, reforçando a importância da rede e apoio familiar para o idoso. A família é a primeira rede social do indivíduo, espaço onde as relações acontecem e se desenvolvem. ${ }^{7}$ Os menores escores foram evidenciados entre os solteiros e viúvos, justificando-se por residirem em famílias multigeracionais, pois estas acabam exercendo um maior domínio sobre o idoso, suas atividades e relacionamentos, enfim, sobre sua vida.

Quanto à atividade remunerada, os idosos que a exercem e que não são aposentados apresentaram melhor qualidade de vida. Ainda é grande o número de idosos que permanece no mercado de trabalho, formal ou informal, que despendem de longas jornadas de trabalho. As atividades informais exigem maiores esforços e acarretam desgastes físicos e mentais; no entanto, permitem ao idoso manter-se ativo e contribuinte para a sociedade. A Tabela 2 apresenta as variáveis e suas conjecturas com as facetas abordadas no instrumento WHOQOL-OLD 
Tabela 2 - Distribuição dos idosos em estudo segundo variáreis sociodemográficas e a proporção de adequação alcançada em cada um dos domínios do WHOQOL-OLD. Foz do Iguaçu, 2010.

\begin{tabular}{|c|c|c|c|c|c|c|c|c|c|c|c|c|c|}
\hline \multirow{2}{*}{ Variáveis } & \multirow[b]{2}{*}{$\mathrm{N}$} & \multicolumn{2}{|r|}{ FS } & \multicolumn{2}{|c|}{ AUT } & \multicolumn{2}{|c|}{ PPF } & \multicolumn{2}{|c|}{ PSO } & \multicolumn{2}{|c|}{ MEM } & \multicolumn{2}{|c|}{ INT } \\
\hline & & $\%$ & $p$ & $\%$ & $p$ & $\%$ & $p$ & $\%$ & $p$ & $\%$ & $p$ & $\%$ & $p$ \\
\hline$\overline{\text { Sexo }}$ & & & 0,2961 & & 0,1591 & & 0,1666 & & 0,0918 & & 0,0977 & & $\overline{1,0000}$ \\
\hline Feminino & 98 & 75,5 & & 64,3 & & 80,6 & & 71,4 & & 81,6 & & 80,6 & \\
\hline Masculino & 94 & 81,9 & & 74,5 & & 88,3 & & 81,9 & & 90,4 & & 80,8 & \\
\hline Idade & & & $<0,0001 *$ & & 0,1947 & & 0,4503 & & 0,2506 & & 0,4486 & & 0,4289 \\
\hline $60-69$ & 97 & 89,7 & & 74,2 & & 87,6 & & 81,4 & & 85,6 & & 81,4 & \\
\hline $70-79$ & 75 & 73,3 & & 66,7 & & 81,3 & & 70,7 & & 84,0 & & 82,7 & \\
\hline$=80$ & 20 & 45,0 & & 55,0 & & 84,2 & & 75,0 & & 95,0 & & 70,0 & \\
\hline Raça/cor & & & 0,4687 & & 0,1036 & & $0,2175^{*}$ & & 0,5984 & & 1,0000 & & $0,0001 *$ \\
\hline Branco & 122 & 80,3 & & 73,8 & & 89,3 & & 77,9 & & 86,1 & & 89,3 & \\
\hline Não branco & 70 & 75,7 & & 61,4 & & 75,7 & & 74,3 & & 85,7 & & 65,7 & \\
\hline Renda & & & 0,0345 & & 0,2342 & & 0,3141 & & 0,6189 & & 0,1829 & & 0,9412 \\
\hline$<1 \mathrm{SM}$ & 18 & 55,5 & & 83,3 & & 83,3 & & 66,7 & & 100 & & 77,8 & \\
\hline 1 a $3 \mathrm{SM}$ & 152 & 80,3 & & 66,4 & & 82,9 & & 75,0 & & 84,9 & & 80,9 & \\
\hline$>3 \mathrm{SM}$ & 22 & 86,4 & & 77,3 & & 95,4 & & 95,4 & & 81,8 & & 81,8 & \\
\hline Religião & & & 0,0853 & & 0,458 & & $0,0268^{*}$ & & 0,5875 & & 0,3189 & & $0,0074 *$ \\
\hline Praticante & 171 & 80,7 & & 70,2 & & 86,5 & & 77,2 & & 84,8 & & 83,6 & \\
\hline Não pratic. & 21 & 61,9 & & 62,0 & & 66,7 & & 71,4 & & 95,2 & & 57,1 & \\
\hline Escolaridade & & & 0,0799 & & $0,0389^{*}$ & & $0,0147^{*}$ & & $0,0244^{*}$ & & 1,0000 & & 0,0694 \\
\hline$=8$ anos & 56 & 87,5 & & 80,4 & & 94,6 & & 87,5 & & 85,7 & & 89,3 & \\
\hline$<8$ anos & 136 & 75,0 & & 64,7 & & 80,1 & & 72,1 & & 86,0 & & 77,2 & \\
\hline Estado Civil & & & $0,0085^{*}$ & & 0,2288 & & 0,08 & & 0,5742 & & 0,8209 & & $0,0080^{*}$ \\
\hline Casado & 136 & 85,3 & & 72,1 & & 87,5 & & 77,9 & & 85,3 & & 86,0 & \\
\hline Não casado & 56 & 62,5 & & 62,5 & & 76,8 & & 73,2 & & 87,5 & & 67,9 & \\
\hline Aposentado & & & 0,0621 & & 0,0279 & & 0,3609 & & 0,5531 & & 0,8096 & & 0,8317 \\
\hline Sim & 146 & 75,3 & & 65,1 & & 82,9 & & 75,3 & & 86,3 & & 80,1 & \\
\hline Não & 46 & 89,1 & & 82,6 & & 89,1 & & 80,4 & & 84,8 & & 82,6 & \\
\hline Atv.Ren & & & $0,0400^{*}$ & & $0,0023 *$ & & $0,0366^{*}$ & & $0,0116^{*}$ & & 0,6646 & & 0,4398 \\
\hline Sim & 64 & 87,5 & & 84,4 & & 92,2 & & 87,5 & & 84,4 & & 84,4 & \\
\hline Não & 128 & 74,2 & & 61,7 & & 80,5 & & 71,1 & & 86,7 & & 78,9 & \\
\hline Reside com & & & 0,5305 & & 0,5604 & & 0,0635 & & 0,1224 & & $0,0416^{*}$ & & 0,4947 \\
\hline Com família & 177 & 79,1 & & 68,4 & & 85,9 & & 78,0 & & 87,6 & & 81,4 & \\
\hline Sozinho & 15 & 73,3 & & 80,0 & & 66,7 & & 60,0 & & 66,7 & & 73,3 & \\
\hline Naturalidade & & & 0,7172 & & 0,001 & & 0,0616 & & $0,0027^{*}$ & & 0,2826 & & 0,1892 \\
\hline Paraná & 70 & 77,1 & & 54,3 & & 91,4 & & 88,6 & & 90,0 & & 75,7 & \\
\hline Outro estado & 122 & 77,0 & & 77,9 & & 80,3 & & 69,7 & & 83,6 & & 83,6 & \\
\hline $\mathbf{N}^{0}$ moradores & & & 0,7945 & & 0,0785 & & 0,1153 & & 0,2861 & & 0,0657 & & $0,0376^{*}$ \\
\hline 1 & 15 & 73,3 & & 80,0 & & 66,7 & & 60,0 & & 66,7 & & 73,3 & \\
\hline 2 & 53 & 81,1 & & 79,2 & & 88,7 & & 77,4 & & 84,9 & & 92,4 & \\
\hline$>2$ & 124 & 78,2 & & 63,7 & & 84,7 & & 78,2 & & 88,7 & & 76,6 & \\
\hline
\end{tabular}


Sentimentos de frustração diante dos anseios da vida que não são realizados e a própria história de cada sujeito marcada por perdas progressivas como a do companheiro, dos laços afetivos, de trabalho, bem como do abandono, do isolamento social e da aposentadoria, que mina os recursos mínimos de sobrevivência, são fatores que comprometem a qualidade de vida do idoso. ${ }^{15}$ Apesar disso, quando abordados sobre conquistas na vida e coisas que se anseiam, os idosos revelaram-se satisfeitos, o que lhes permite uma qualidade de vida satisfatória. A interpretação que este idoso faz da relação entre seu passado e presente pode ser marcado por um contexto socioeconômico. Assim, embora as condições de vida não tenham mudado significativamente, é fato que hoje eles não enfrentam mais as exigências culturais e sociais decorrentes de atividades como cuidar dos filhos e ser responsável pela manutenção da família.

A participação social reduz com o passar da idade, dado evidenciado especialmente quando os idosos ultrapassam os 80 anos, pois nesta fase as limitações próprias do processo de envelhecimento estão presentes de forma mais contundente, fazendo com que eles permaneçam maior tempo junto à família. Contudo, é interessante observar, que as mulheres se destacam na participação social, alcançando escores mais elevados, o que demonstra que elas estão mais abertas e disponíveis para um maior envolvimento em atividades fora do domicílio, na comunidade, em grupos de convivência, espaços em que a participação feminina é significativamente maior.

A morte constitui um processo natural da existência humana, juntamente com o envelhecer, porém nem sempre são aceitos pelos seres que o vivenciam. Quando há uma valorização do saber e da história de vida de cada idoso, quando estes são entendidos e respeitados, ele percebe que sua existência tem um significado. Considerar, respeitar e amar permite 0 acolhimento ao idoso, o reconhecimento de sua singularidade, promovendo um sentido no envelhecer e possibilitando 0 enfrentamento da morte. ${ }^{16}$

A faceta Intimidade foi a única que revelou ser maior no sexo feminino. Mulheres idosas experimentam uma maior probabilidade de ficarem viúvas, fazendo com que as relações pessoais e íntimas sejam estimuladas com outras pessoas. Essa viuvez pode significar autonomia e liberdade.

\section{CONSIDERAÇÕES FINAIS}

A maioria dos idosos selecionados tinha entre 60 a 69 anos, morava com a família, era casada, seguia uma religião, tinha baixa escolaridade e baixa renda. As características, portanto, são bastante semelhantes às características da população brasileira e podem influenciar negativamente em sua QV.

Os déficits de habilidades sensoriais apesar de presentes nos idosos, não são muitas vezes percebidas, não trazendo impacto direto em sua QV até que esta se torne evidente, necessitando de intervenção médica e uso de próteses. Essas limitações parecem não afetar a percepção subjetiva do idoso com sua saúde, porém devem ser valorizadas pelos profissionais de saúde, com vistas a retardar suas manifestações.

A importância da rede familiar para o idoso ficou evidente, sendo demonstrada como rede de apoio, iniciando desde o nascimento, passando pela adolescência até a vida adulta. A forma como ele vivenciou este processo será repassado para outras gerações, embora ele seja, independente da utilidade econômica, política ou social, um serúnico.

Entretanto, nem toda a família está preparada para receber um idoso debilitado, pois ainda encontramos idosos que vivem sós. Alguns formam novos elos, mas a falta da estrutura familiar é muito marcante, sendo um fato que interfere na qualidade de vida.

As mulheres idosas demonstram uma participação social muito forte e ativa ao assumirem papéis não esperados, como agentes de transformação da sociedade, como chefes de famílias, como principais cuidadoras e como fonte principal de renda.

Embora as mulheres vivam mais do que os homens, elas passam por um período maior de debilitação biológica antes da morte do que no sexo masculino, o que também precisa ser considerado de forma contundente pelos profissionais de saúde.

A percepção do idoso da morte ficou clara perante os altos escores observados, demonstrando-se como um fato natural, assim como o envelhecimento.

A questão social do idoso aliada à complexidade da vida exigem medidas urgentes no sentido de atingir o objetivo da Política Nacional de Saúde do Idoso, que é permitir um envelhecimento saudável, preservando sua capacidade funcional, sua autonomia e mantendo o nível de qualidade de vida.

A QV tem uma grande abrangência de significados e valores e depende de diversos fatores como os sociais, os ambientais, econômicos, biológicos e psíquicos, ou seja, de acordo com que o idoso vivencia ou vivenciou ao longo da vida. 0 objetivo do estudo foi identificar fatores que pudessem influenciar na QV, referentes às diferentes facetas propostas que integram o WHOQOL-OLD e, desta forma, buscar intervenções de saúde para um envelhecimento de maior qualidade.

0 instrumento utilizado mostrou-se útil para avaliação da qualidade de vida, porém, não o suficiente para explicar a grande variabilidade de significados que o idoso pode revelar, 
expondo outros fatores significantes. A avaliação de um mesmo indivíduo pode variar com o tempo, com as prioridades ao longo da vida e com as modificações desta com o tempo, sendo multifatorial e multidimensional, fato que merece destaque para o delineamento de novas pesquisas.

Melhorar a qualidade de vida desta parcela da população, que tende a crescer nos próximos anos, é de grande valia social. 0 enfermeiro e a equipe de saúde devem pensar no gerenciamento de ações, levando orientações, suporte informal e familiar que atendam as necessidades espećficas do idoso. A sugestão é que sejam realizadas mais pesquisas, em especial com idosos saudáveis, com o objetivo de compreendê-los para que as propostas não se percam, e que a qualidade de vida seja evidenciada.

\section{REFERÊNCIAS}

1. Veras R. Envelhecimento populacional e as informações de saúde do PNAD: demandas e desafios contemporâneos. Cad Saude Publica 2007 out; 23(10): 2463-66.

2. Instituto Brasileiro de Geografia e Estatística-IBGE. Dados preliminares do censo 2000. Disponível em: http://www.ibge.com.br.

3. Linck CL, Lange C, Schwartz E, Dilélio AS, Zillmer JGV, Thorferhn MB. A inserção do idoso no contexto da pós-modernidade. Cienc Cuid Saude 2009 dez; 8 (supl):130-35.

4. Parahyba MI, Simões CCS. A prevalência de incapacidade funcional em idosos no Brasil. Cienc Saude Colet 2006; 11: 967-74.

5. Rodrigues RAP, Diogo MJD. Como cuidar dos idosos. $4^{\mathrm{a}}$ ed. São Paulo (SP): Papirus; 2004.

6. Meireles VC, Matsuda LM, Coimbra JAH, Mathias TAF. Característica dos idosos em área de abrangência do Programa Saúde da Família na região noroeste do Paraná: contribuição para a gestão do cuidado em enfermagem. Saude Soc 2007 abr; 16 (1): 69-80.

7. Marcon SS, Carreira L, Waidman MAP, Andrade OG. 0 cotidiano do idoso e suas relações familiares revelando indícios de qualidade de vida. Texto\&Contexto Enfem 1999 dez; 8(3): 200-12.

8. Campo MO, Rodrigues Neto JFR. Qualidade de vida, um instrumento para promoção da saúde. Rev Baiana Saude Publica. 2008 ago; 32(2): 232-40.

9. Pereira RJ, Cotta RMM, Franceschini SCC, Ribeiro RCL, Sampaio RF, Priore SE, et al. Contribuição dos domínios físico, social, psicológico e ambiental para a qualidade de vida global de idosos. Rev Psiquiatr Rio Gd Sul [periódico na Internet]. 2006; 28(1):[aprox 12 p.]. Disponível em: <http:/ /www.revistapsiqrs.org.br>

10. Fleck MP, Chachamovich E, Trentini C. Development and validation of the Portuguese version of the WHOQOL-Old module. Rev Saude Publica 2006; 40(5):785-91
11. Santos SSC. Enfermagem gerontogeriátrica: reflexão à ação cuidativa. $2^{\mathrm{a}}$ ed. São Paulo (SP): Robe; 2001.

12. Sousa AI, Silver LD. Perfil sociodemográfico e estado de saúde autoreferido entre idosas de uma localidade de baixa renda. Esc Anna Nery. 2008 dez; 12(4): 706-16.

13. Fleck MPA, Louzada S, Xavier M, Chachamovich E, Vieira G, Santos L, et al. Aplicação da versão em português do instrumento abreviado de avaliação da qualidade de vida "WHOQOL-Bref". Rev Saude Publica. 2000; 34(2): 178-83.

14. Instituto Brasileiro de Geografia e Estatística-IBGE. Tábuas Completas de Mortalidade - 2003. Disponível em: http://www.ibge.gov.br/home/ presidencia/noticias/noticia_visualiza.php?id_noticia=266\&id_pagina $=1$.

15. Pacheco JL. Educação, trabalho e envelhecimento: estudo das histórias de vida de trabalhadores assalariados e suas relações com a escola, com o trabalho e com os sintomas depressivos, após a aposentadoria [tese]. Campinas (SP): UNICAMP; 2002.

16. Frumi C, Celich KLS. 0 olhar do idoso frente ao envelhecimento e à morte. Rev Bras Cienc Envelh Hum 2006 dez; 3(2): 92-100.

17. Teixeira RA, Freitas CR, Millão LF, Gonçalves AK, Junior BB, Santos AMPV, et al. Relação entre a queixa e a presença de perda auditiva entre idosos. Arq Int. Otorrinolaringol. 2009 fev; 13(1): 78-82.

18. Ministério da Saúde (BR). Lei 10.741, de 01 de outubro de 2003. Dispõe sobre o Estatuto do Idoso e dá outras providências. Disponível em: http://www.planalto.gov.br/ccivil-03/leis/2003/L10.741.html. 